Bundesgesundheitsbl 2015 · 58:1378-1389

DOI 10.1007/s00103-015-2252-0

Online publiziert: 20. Oktober 2015

(c) Springer-Verlag Berlin Heidelberg 2015
Bekanntmachung des Umweltbundesamtes

\section{Richtwerte für Dimethylbenzole in der Innenraumluft}

\section{Mitteilung des Ausschusses für Innenraumrichtwerte}

\section{Stoffidentifikation [1]}

\begin{tabular}{|c|c|c|c|c|}
\hline $\begin{array}{l}\text { Rationeller } \\
\text { Name }\end{array}$ & $\begin{array}{l}\text { 1,2-Dimethyl- } \\
\text { benzol }\end{array}$ & $\begin{array}{l}\text { 1,3-Dimethyl- } \\
\text { benzol }\end{array}$ & $\begin{array}{l}\text { 1,4-Dimethyl- } \\
\text { benzol }\end{array}$ & Dimethylbenzole \\
\hline Synonyme & $\begin{array}{l}\text { ortho-Xylol } \\
\text { 2-Methyltoluol }\end{array}$ & $\begin{array}{l}\text { meta-Xylol } \\
\text { 3-Methyltoluol }\end{array}$ & $\begin{array}{l}\text { para-Xylol } \\
\text { 4-Methyltoluol }\end{array}$ & $\begin{array}{l}\text { Xylole } \\
\text { Methyltoluole }\end{array}$ \\
\hline CLP-Index-Nr. & $601-038-00-6$ & $601-039-00-1$ & $601-040-00-7$ & $601-022-00-9$ \\
\hline EG-Nr. & $202-422-2$ & $203-576-3$ & 203-396-5 & $215-535-7$ \\
\hline CAS-Nr. & $95-47-6$ & $108-38-3$ & $106-42-3$ & $1330-20-7$ \\
\hline Summenformal & $\mathrm{C}_{8} \mathrm{H}_{10}$ & $\mathrm{C}_{8} \mathrm{H}_{10}$ & $\mathrm{C}_{8} \mathrm{H}_{10}$ & $\mathrm{C}_{8} \mathrm{H}_{10}$ \\
\hline Strukturformel & $\mathrm{CH}_{\mathrm{H}}$ & & $\mathrm{CH}_{3}$ & \\
\hline
\end{tabular}

\subsection{Physikalische und chemische Eigenschaften [2-4]}

\begin{tabular}{|llll}
\hline & \multicolumn{3}{c}{ Xylol-lsomer } \\
\cline { 2 - 4 } & ortho-Xylol & meta-Xylol & para-Xylol \\
\hline Molmasse $(\mathrm{g} / \mathrm{mol})$ & 106,17 & 106,17 & 106,17 \\
\hline Schmelzpunkt $\left({ }^{\circ} \mathrm{C}\right)$ & $-25,2$ & $-47,9$ & 13,3 \\
\hline Siedepunkt bei $1013 \mathrm{hPa}\left({ }^{\circ} \mathrm{C}\right)$ & 144,4 & 139,1 & 138,3 \\
\cline { 2 - 4 } & \multicolumn{1}{c}{ Technisches Xylol: $137-140$} \\
\hline Dampfdruck (hPa) & $8,8\left(25^{\circ} \mathrm{C}\right)$ & $11,1\left(25^{\circ} \mathrm{C}\right)$ & $11,7\left(25^{\circ} \mathrm{C}\right)$ \\
\cline { 2 - 4 } & Technisches Xylol: $7-9\left(20^{\circ} \mathrm{C}\right)$ \\
\hline Relative Gasdichte $(\mathrm{Luft}=1)$ & 3,7 & 3,7 & 3,7 \\
\hline Wasserlöslichkeit bei $25^{\circ} \mathrm{C}(\mathrm{mg} / \mathrm{l})$ & 170 & 146 & 156 \\
\hline Verteilungskoeffizient $\operatorname{lg~} \mathrm{K}$ Octanol/Wasser & 3,12 & 3,2 & 3,15 \\
\hline Umrechnung $\left(20^{\circ} \mathrm{C}\right)$ & $1 \mathrm{ml} / \mathrm{m}^{3}=4,4 \mathrm{mg} / \mathrm{m}^{3}, 1 \mathrm{mg} / \mathrm{m}^{3}=0,23 \mathrm{ml} / \mathrm{m}^{3}$ \\
\hline
\end{tabular}

\subsection{Stoffeigenschaften und Anwendung}

Dimethylbenzole zählen zu den aromatischen Kohlenwasserstoffen und bestehen jeweils aus einem Benzolring mit zwei Methylsubstituenten. Durch die unterschiedliche Anordnung der Methylgruppen am Ring ergeben sich drei Konstitutionsisomere. Technische Dimethylbenzol-Gemische, die aus Öl gewonnen werden, enthalten in der Regel etwa $45 \%$ mXylol und jeweils etwa $20 \%$ o- und p-Xylol sowie etwa $15 \%$ Ethylbenzol, das im gleichen Temperaturbereich siedet. Aus Steinkohlenteer hergestelltes technisches Xylol enthält geringere Anteile an o-Xylol und Ethylbenzol und einen höheren Anteil von 45-70\% m-Xylol [4]. In geringeren Anteilen können in technischem Xylol außerdem Toluol und $\mathrm{C}_{9}$-Alkylbenzole enthalten sein [1].

Alle drei Xylole und das technische Gemisch sind bei Raumtemperatur farblose Flüssigkeiten mit süßlichem Geruch nach „aromatischem Lösemittel“ [5]. Zusammen mit Benzol, Toluol und Ethylbenzol bilden Xylole die Gruppe der BTXE-Aromaten, die großtechnische Bedeutung besitzen. Xylole finden als Isomerengemisch, meist ohne Abtrennung des Ethylbenzols, Verwendung als Lösemittel, etwa in Farben und Farbverdünnern, aber auch bei der Vorbereitung von Gewebsproben für histologische Untersuchungen sowie als Zusatz in Treibstoffen [3]. Die jeweiligen einzelnen Isomere dienen als Ausgangsstoff zur Herstellung anderer organischer Verbindungen wie Phthalsäureanhydrid (aus o-Xylol), Isophthalsäure 


\begin{tabular}{|c|c|c|c|c|c|c|}
\hline Innenraum/Studie & $N$ & $B G\left(\mu g / m^{3}\right)$ & $N>B G(\%>B G)$ & $\begin{array}{l}\text { Median } \\
\left(\mu \mathrm{g} / \mathrm{m}^{3}\right)\end{array}$ & $\begin{array}{l}\text { 95. Perzentil } \\
\left(\mu \mathrm{g} / \mathrm{m}^{3}\right)\end{array}$ & $\begin{array}{l}\text { Maximum } \\
\left(\mu \mathrm{g} / \mathrm{m}^{3}\right)\end{array}$ \\
\hline \multirow[t]{2}{*}{ n.a./B.A.U.C.H. 1989-1999 [10] } & $\mathrm{m} / \mathrm{p}: 700$ & n.a. & n.a. & 8 & 84 & n.a. \\
\hline & o: 700 & n.a. & n.a. & 2 & 30 & n.a. \\
\hline \multirow[t]{2}{*}{ n.a./GFU 1995-2000 [10] } & $\mathrm{m} / \mathrm{p}: 771$ & n.a. & n.a. & 9 & 76 & n.a. \\
\hline & o: 771 & n.a. & n.a. & 3 & 27 & n.a. \\
\hline \multirow[t]{2}{*}{ Umwelt-Survey 1985/86 [11] } & $\mathrm{m} / \mathrm{p}: 479$ & n.a. & n.a. & 16 & 57 & n.a. \\
\hline & o: 479 & n.a. & n.a. & 5 & 18 & n.a. \\
\hline \multirow[t]{3}{*}{ Wohnungen, KUS 2003-2006 [12] } & o/m/p: 555 & n.a. & n.a. & 4,5 & 21 & 248 \\
\hline & $\mathrm{m} / \mathrm{p}: 555$ & 1 & $503(91)$ & 3,2 & 16 & 200 \\
\hline & o: 555 & 1 & $314(57)$ & 1,2 & 5,5 & 48 \\
\hline \multirow[t]{2}{*}{ Wohn- und Büroräume/RKI 1999-2003 [10] } & $\mathrm{m} / \mathrm{p}: 52$ & 1 & $52(100)$ & 5 & 18 & n.a. \\
\hline & $0: 52$ & 1 & $47(96)$ & 2 & 8 & n.a. \\
\hline Büroräume 2001-2005 [13] & $429^{a}$ & n.a. & n.a. & 8 & n.a. & n.a. \\
\hline \multirow[t]{4}{*}{ Innenräume, v. a. Büros 2006-2010 [14] } & $72^{\mathrm{a}}$ & 5 & $32(44)$ & n.a. & 39 & n.a. \\
\hline & o: 977 & 10 & $816(84)$ & n.a. & $<10$ & n.a. \\
\hline & m:991 & 10 & $565(57)$ & n.a. & 26 & n.a. \\
\hline & p: 992 & 10 & $832(84)$ & n.a. & $<10$ & n.a. \\
\hline \multirow[t]{2}{*}{ Büro, Wohnung, Schule, Kita u. a./AGÖF 2008 [15] } & $\mathrm{m} / \mathrm{p}: 2396$ & 1 & $2305(96)$ & 5 & 86 & 6155 \\
\hline & $0: 2375$ & 1 & $1994(84)$ & 2 & 29 & 2156 \\
\hline \multirow[t]{2}{*}{ Büroräume/AGÖF, 2002-2006 [16] } & m/p: 943 & 1 & $920(98)$ & 5,5 & 85 & 6155 \\
\hline & o: 939 & 1 & $820(87)$ & 2 & 29 & 2156 \\
\hline \multirow[t]{2}{*}{ Klassenzimmer/AGÖF, 2002-2006 [16] } & m/p: 282 & 1 & $260(92)$ & 3 & 389 & 1380 \\
\hline & o: 280 & 1 & $200(71)$ & 1 & 13 & 400 \\
\hline \multirow[t]{2}{*}{ Schlaf- und Wohnzimmer/AGÖF, 2002-2006 [16] } & $\mathrm{m} / \mathrm{p}: 617$ & 1 & $591(96)$ & 5 & 76 & 1363 \\
\hline & o: 609 & 1 & $501(82)$ & 2 & 23 & 468 \\
\hline Klassenzimmer/AGÖF, 2006-2012 [17] & m/p:n.a. & 1 & n.a. & n.a. & 22 & n.a. ${ }^{b}$ \\
\hline Wohnräume/AGÖF, 2006-2012 [17] & m/p: n.a. & 1 & n.a. & n.a. & 9,9 & n.a. ${ }^{\text {b }}$ \\
\hline Schulen und Kindergärten, Schl.-Holst. 1990-93 [18] & $\mathrm{m} / \mathrm{p}: 395$ & n.a. & n.a. & 4,5 & 68 & n.a. \\
\hline Kindergärten, Berlin, 2000/2001 [18] & $\mathrm{m} / \mathrm{p}: 26$ & n.a. & $26(100)$ & 6,9 & 31 & n.a \\
\hline Klassenräume, Berlin, 2002/2003 [19] & m/p: 39 & n.a. & $39(100)$ & 2,5 & 9,3 & n.a. \\
\hline Klassenräume, Bayern, Winter 2005 [18] & m/p: 90 & 0,1 & $90(100)$ & 3,0 & 9,3 & n.a. \\
\hline \multirow[t]{3}{*}{ Schulen und Kindergärten, Schl.-Holst. 2005-2007 [20] } & $0: 285$ & 2 & $176(62)$ & 1 & 5,7 & 70 \\
\hline & $\mathrm{m}: 285$ & 2 & $209(73)$ & 1 & 13 & 130 \\
\hline & p: 285 & 2 & $154(54)$ & 1 & 9 & 79 \\
\hline \multirow[t]{2}{*}{ Klassenzimmer ohne Schüler, NRW 2013 [21] } & o: 280 & 1 & $136(49)$ & 1 & 5 & n.a. \\
\hline & $\mathrm{m} / \mathrm{p}: 280$ & 1 & $208(74)$ & 2 & 15 & n.a. \\
\hline
\end{tabular}

(aus m-Xylol) und Terephthalsäure (aus p-Xylol), die zu Kunststoffen, Farbstoffen, Insektiziden und pharmazeutischen Produkten weiterverarbeitet werden [3].

\section{Exposition}

Eine Exposition der Allgemeinbevölkerung erfolgt in erster Linie gegenüber gasförmigen Xylolen über die Atemwege, daneben kann es bei der Verwendung entsprechender Produkte zur Aufnahme in flüssiger Form über die Haut kom- men. Als ubiquitäre Hintergrundkonzentration in der Außenluft, die im Wesentlichen aus der Produktion, Distribution und Verwendung (Raffinerien, Tankstellen, Verkehr) in Treibstoffen stammt, werden Werte von $0,1 \mu \mathrm{g} / \mathrm{m}^{3}$ für ländliche Gebiete und bis zu $50 \mu \mathrm{g} / \mathrm{m}^{3}$ für Ballungsräume angegeben [6]. Aktuelle, in mehreren Städten in Hessen durchgeführte Messungen erbrachten Jahresmittelwerte an $\mathrm{m}$ - $/$ p-Xylol von 1,09-3,32 $\mu \mathrm{g} / \mathrm{m}^{3}$ [7]. Ähnliche Konzentrationen von $0,48-3,1 \mu \mathrm{g} / \mathrm{m}^{3}$ $\mathrm{m}$-/p-Xylol sowie $0,14-0,95 \mu \mathrm{g} / \mathrm{m}^{3} \mathrm{o}$-Xylol wurden auch in neueren Untersuchungen in bayerischen Städten ermittelt [8].

In Lebensmitteln wurden Xylole in geringer Konzentration (3-9 $\mu \mathrm{g} / \mathrm{kg}$ Trockengewicht) nachgewiesen, in anderen Untersuchungen wurde das Vorkommen nicht quantifiziert [9]. Es liegen keine Angaben darüber vor, ob Xylole als natürliche Bestandteile in Lebensmitteln auftreten können. 


\subsection{Innenraumluft}

Zum Vorkommen von Xylolen in der Innenraumluft von Wohnungen, Büroräumen sowie Schulen und Kindergärten liegen einige Angaben vor (• Tab. 1). In den Messungen wurden $\mathrm{m}$ - und $\mathrm{p}$-Xylol meist nicht getrennt bestimmt. Beide Stoffen wurden fast immer gefunden [15]; in etwa $80 \%$ aller untersuchten Innenräume wurde auch o-Xylol nachgewiesen.

Die 95. Perzentile der neuesten Untersuchungen in Schulen und Kindertagesstätten weisen auf einen Rückgang der Xylole-Immissionen gegenüber früheren Untersuchungen hin. Damit zeigt sich bei den Xylolen ein ähnlicher Trend wie bei Ethylbenzol und höheren Alkylbenzolen $[22,23]$.

\subsection{Gesamtexposition}

Eine in den 1990ern von der kanadischen Gesundheitsbehörde vorgenommene Abschätzung ergab eine Gesamtbelastung der Allgemeinbevölkerung durch Xylole in der Außen- und Innenraumluft, beim Tanken, im Trinkwasser sowie durch Zigarettenrauch von 3,7-9,5 $\mu \mathrm{g} / \mathrm{d}$. Aufgeschlüsselt nach Altersgruppen ergab sich die höchste, auf das Körpergewicht (KG) bezogene Belastung mit 0,46-0,89 $\mu$ g Xylole/kg KG und Tag für Säuglinge bis zu einem Alter von einem halben Jahr [24]. Neuere Abschätzungen liegen nicht vor.

\section{Toxikokinetik}

\subsection{Aufnahme und Verteilung}

Xylole werden inhalativ sowie - in flüssiger Form - oral und auch dermal gut resorbiert. Eine Aufnahme von gasförmigen Xylolen über die Haut spielt neben der Aufnahme über die Atemwege für die Gesamtexposition hingegen keine nennenswerte Rolle (ca. 0,2\%) [25]. Aus den in $\bullet$ Tab. 2 angeführten Werten ergibt sich, dass bei inhalativer Exposition etwa $60-70 \%$ über die Atemwege aufgenommen werden. Mit sinkender Konzentration in der Einatemluft nimmt die Retention zu und erreicht bei $100 \mathrm{mg}$ Xylole/ $\mathrm{m}^{3}$ etwa $85 \%$. Zwischen den drei Isomeren wurden keine Unterschiede in der inhalativen Aufnahme festgestellt.

Bundesgesundheitsbl 2015 · 58:1378-1389 DOI 10.1007/s00103-015-2252-0

c) Springer-Verlag Berlin Heidelberg 2015

Bekanntmachung des Umweltbundesamtes

Richtwerte für Dimethylbenzole in der Innenraumluft. Mitteilung des Ausschusses für Innenraumrichtwerte

\section{Zusammenfassung}

Zum Schutz der Gesundheit der Bevölkerung setzt der Ausschuss für Innenraumrichtwerte Richtwerte für die Innenraumluft fest. Für eine gesundheitliche Bewertung von Xylolen in der Luft liegen keine hinreichend aussagekräftigen Humanstudien vor. In einer als zuverlässig bewerteten subchronischen Inhalationsstudie mit $\mathrm{m}$-Xylol an männlichen Ratten wurden neurotoxische Wirkungen beobachtet, die sich in einer Beeinträchtigung der neuromuskulären Koordination im Rotarod-Test zeigten. Aus dieser Studie ergibt sich umgerechnet auf eine kontinuierliche Exposition eine LOAEC von $39 \mathrm{mg} \mathrm{Xylol} / \mathrm{m}^{3}$ für den
Endpunkt Neurotoxizität. Mit einem Extrapolationsfaktor von 2,5 für Interspeziesunterschiede, von 10 für interindividuelle Variabilität sowie einem Faktor von 2 zur Berücksichtigung der im Vergleich mit Erwachsenen höheren Atemrate von Kindern leitet der Ausschuss einen Richtwert II (Gefahrenrichtwert) von $0,8 \mathrm{mg} / \mathrm{m}^{3}$ Xylol $/ \mathrm{m}^{3}$ und einen Richtwert I (Vorsorgerichtwert) von $0,1 \mathrm{mg} \mathrm{Xylol} / \mathrm{m}^{3}$ Raumluft ab.

Schlüsselwörter

Xylol · Dimethylbenzol · Innenraumluft . Neurotoxizität · Richtwert

\section{Indoor air guide values for dimethylbenzene. Communication from the Committee on Indoor air guide values}

\section{Abstract}

The German Committee on Indoor Guidelines is issuing indoor air guide values to protect public health. No sufficiently valid human studies are available for the health evaluation of xylenes in indoor air. In a validated subchronic inhalation animal study on $\mathrm{m}$-xylene using male rats, neurotoxic effects were observed, which led to an decreased neuromuscular coordination as observed in a rotarod test. Conversion of experimental to continuous exposure leads to a LOAEC of $39 \mathrm{mg}$ xylene $/ \mathrm{m}^{3}$ for the endpoint neurotox- icity. A health hazard guide value (RW II) of $0.8 \mathrm{mg}$ xylene $/ \mathrm{m}^{3}$ is derived by applying an interspecies factor of 2.5, a factor of 10 for interindividual variability, and a factor of 2 to account for the higher respiratory rate of children compared to adults. A health precaution guide value of $0.1 \mathrm{mg}$ xylene $/ \mathrm{m}^{3}$ is recommended.

\section{Keywords}

Xylene · Dimethylbenzene · Indoor air .

Neurotoxicity · Guide value
Bei oraler Exposition wird Xylol rasch und weitestgehend resorbiert [1].

Experimentell ermittelte Blut-LuftVerteilungskoeffizienten ergeben für o-, m- und p-Xylol Werte von 44,3, 41,3 und 46,0 (Rattenblut) bzw. 35,2, 31,9 und 39,0 (Humanblut) [31]. In den Körper aufgenommene Xylole werden aus dem Blut rasch in alle Organe verteilt. Untersuchungen am Menschen ergaben, dass 10$20 \%$ der aufgenommenen Xylole in das Fettgewebe verteilt werden [27]. In vitro-Untersuchungen mit o-, $\mathrm{m}$ - und $\mathrm{p}$-Xylol an Geweben unterschiedlicher Organe von Ratten ergaben Gewebe-Blut-Verteilungskoeffizienten von 1,5-3,7 für Hirn, Muskel und Niere, von 3,2-5,7 für Lebergewebe und von 37 bis 67 für Fettgewebe [32]. In Übereinstimmung mit diesem Be- fund erreichte die Konzentration im Gewebe von Ratten nach einer Exposition bis zu $4840 \mathrm{mg} \mathrm{m-Xylol} / \mathrm{m}^{3}$ den höchsten Wert im Fettgewebe, in abnehmender Konzentration gefolgt von Leber, Niere und Hirn [33, 34]. Vergleichbare Ergebnisse liegen an Mäusen vor $[35,36]$. Tierexperimentelle Untersuchungen mit ound p-Xylol belegen, dass Xylole die Plazentaschranke überwinden und auch in die Milch übergehen können [31].

\subsection{Metabolismus und Ausscheidung}

Wie bei anderen Alkylbenzolen erfolgt auch die Metabolisierung von Xylolen durch mikrosomale P450-Monoxygenasen. Der Hauptweg besteht in der Oxi- 


\begin{tabular}{|c|c|c|c|}
\hline Exposition & $\begin{array}{l}\text { Pulmonale } \\
\text { Retention }\end{array}$ & Anmerkung & Ref. \\
\hline $200-400 \mathrm{mg} / \mathrm{m}^{3}, 8 \mathrm{~h}$ & $62-64 \%$ & $\begin{array}{l}\text { Vergleichbare Werte für alle drei } \\
\text { Isomere }\end{array}$ & [26] \\
\hline $\begin{array}{l}434-868 \mathrm{mg} / \mathrm{m}^{3}, \\
2 \times 3 \mathrm{~h}\end{array}$ & $60 \%$ & $\begin{array}{l}\text { Xylole-Gemisch, vergleichbarer } \\
\text { Wert auch bei zusätzlichen } \\
\text { Expositionsspitzen }\end{array}$ & {$[27]$} \\
\hline $400 \mathrm{mg} / \mathrm{m}^{3}, 8 \mathrm{~h}$ & $58 \%$ & m-Xylol & [28] \\
\hline $\begin{array}{l}434-868 \mathrm{mg} / \mathrm{m}^{3}, \\
4 \times 30 \mathrm{~min}\end{array}$ & $\begin{array}{l}65 \% \text { (in Ruhe oder } \\
\text { leicht aktiv) } \\
50 \% \text { (körperlich aktiv) }\end{array}$ & Xylole-Gemisch & [29] \\
\hline $100 \mathrm{mg} / \mathrm{m}^{3}, 8 \mathrm{~h}$ & $(84 / 87 \%)$ & \multirow{3}{*}{$\begin{array}{l}\text { m-Xylol, Mittelwert (Anfangs-I } \\
\text { Endwert) }\end{array}$} & \multirow[t]{3}{*}{ [30] } \\
\hline $300 \mathrm{mg} / \mathrm{m}^{3}(8 \mathrm{~h})$ & $75 \%(83 / 67 \%)$ & & \\
\hline $600 \mathrm{mg} / \mathrm{m}^{3}(8 \mathrm{~h})$ & $71 \%(78 / 65 \%)$ & & \\
\hline
\end{tabular}

dation einer der beiden Methylgruppen unter Bildung des o-, m- bzw. p-Methylbenzylalkohols, der in nachfolgenden Schritten zur entsprechenden o-, mund p-Methylbenzoesäure weiteroxidiert wird. Eine Oxidation im Ring unter Bildung von Dimethylphenolen (Xylenolen) findet nur in untergeordnetem Ausmaß statt. Die auf dem Hauptweg gebildete Carbonsäure wird nach Konjugation mit Glycin zu o-, m- bzw. p-Methylhippursäure mit dem Urin ausgeschieden. Der genannte Abbauweg stellt sowohl beim Menschen als auch bei der Ratte den Hauptweg dar. In geringerer Menge kann auch eine Konjugation der Carbonsäure mit Glukuronsäure erfolgen, die gebildeten Glucuronide werden ebenfalls renal eliminiert.

In Untersuchungen mit kontrollierter inhalativer Exposition von Probanden wurden nur $5 \%$ des absorbierten $\mathrm{Xy}$ lole-Gemisches nachfolgend unverändert wieder abgeatmet, $95 \%$ hingegen als Methylhippursäuren ausgeschieden [27]. In einer anderen Untersuchung mit inhalativer Exposition von Probanden gegenüber den jeweiligen einzelnen Isomeren zeigten sich nur geringe Unterschiede im Ausmaß der Metabolisierung: 5,3, 5,8 bzw. 3,5\% des absorbierten o-, $\mathrm{m}$ - und p-Xylols wurden unverändert wieder abgeatmet, $97,1,99,2$ und $95,1 \%$ der metabolisierten Menge wurden in Form der entsprechenden Methylhippursäure renal eliminiert, geringe Anteile von 0,86, 1,98 bzw. 0,05\% des metabolisierten o-, $\mathrm{m}$ - und p-Xylols entfielen auf Dimethylphenole [26]. Nach inhalativer Exposition gegenüber $220 \mathrm{mg} \mathrm{m}$-Xylol $/ \mathrm{m}^{3}$ zeig- ten sich nur geringfügige Unterschiede im Ausmaß der Ausscheidung von Methylhippursäure zwischen Männern und Frauen [37].

Die Elimination von Xylolen lässt sich durch eine biphasische Kinetik mit einer schnellen Phase (Halbwertszeit etwa $1 \mathrm{~h}$ ) und einer langsameren (Halbwertszeit etwa $20 \mathrm{~h}$ ) beschreiben [31]. Als terminale Halbwertszeit wird für alle Isomere ein durchschnittlicher Wert von $34 \mathrm{~h}$ angegeben, wobei die Werte für einzelne Individuen von 12 bis $108 \mathrm{~h}$ reichten [38].

Die Ausscheidung der Methylhippursäuren wird zur Beurteilung der inneren Belastung nach einer Exposition gegenüber Xylolen am Arbeitsplatz verwendet [39].

\section{Wirkungen}

Den wesentlichen Wirkungsendpunkt bei einer Exposition gegenüber Xylolen bilden Beeinträchtigungen des zentralen Nervensystems. Insbesondere bei kurzzeitiger Einwirkung wurden außerdem Reizeffekte auf die Schleimhäute des Atemtrakts und die Augen beschrieben. Die Ergebnisse dieser Studien wurden in Übersichtsarbeiten zusammengefasst $[4,9,40-43]$.

\subsection{Irritative Wirkungen}

In einer Untersuchung auf Reizwirkungen klagten von 50 Probanden, die $30 \mathrm{~min}$ gegenüber $0,440,880$ oder 1760 mg Xylole $/ \mathrm{m}^{3}$ exponiert waren, 60,70 bzw. $90 \%$ über Augenreizungen, aber auch die Hälf- te der Kontrollgruppe gab Augenreizungen an [44].

In einer weiteren Untersuchung wurden je 28 Männer und Frauen $2 \mathrm{~h}$ lang gegenüber $220 \mathrm{mg} \mathrm{m}$-Xylol $/ \mathrm{m}^{3}$ exponiert [45]. Es wurden sowohl subjektive Eindrücke (anhand einer visuellen Analogskala) als auch objektive Parameter wie Lungenfunktion, nasale Schleimhautschwellung, Entzündungsmarker in der nasalen Lavage und die Fähigkeit zur Farbdifferenzierung untersucht. Für nahezu alle der zehn abgefragten Symptomparameter (Unbehagen in Augen, Nase, Rachen, Atembeschwerden, Kopfschmerzen, Schwindelgefühl, Rauscheindruck) wurden nach einer Stunde oder am Ende der Exposition leicht erhöhte Einschätzungen gegeben. Die meisten Veränderungen waren in beiden Geschlechtern $\mathrm{zu}$ mindestens einem der beiden Zeitpunkte im Vergleich zur Kontrollexposition ohne Xylol signifikant; Frauen berichten insbesondere Unbehagen in Rachen und Atemwegen etwas häufiger als Männer. Müdigkeit und Übelkeit traten nicht auf. In der Gesamteinschätzung wurde die Expositionskonzentration von $220 \mathrm{mg} / \mathrm{m}^{3}$ als "minimale LOAEC“ eingestuft. Lungenfunktionswerte (FVC, $\mathrm{FEF}_{75}$ ) waren bei Frauen $3 \mathrm{~h}$ nach Expositionsende minimal $(<4 \%)$ vermindert, alle anderen Parameter waren bei Männern und Frauen unmittelbar im Anschluss an die Exposition und $3 \mathrm{~h}$ später nicht verändert [45].

Die sensorische Wirkung von Xylolen, die durch eine Reizung des Trigeminus der Nasenschleimhaut verursacht wird, wurde an Swiss-Mäusen anhand der Abnahme der Respirationsrate und der Veränderungen von Atemvolumen und exspiratorischer Flussrate untersucht (Alarie-Test). Nach kurzfristiger Einwirkung wurde eine Verminderung der Atemrate um $50 \%\left(\mathrm{RD}_{50}\right)$ bei $6460 \mathrm{mg}$ o-Xylol/ $\mathrm{m}^{3}$ bzw. $5830 \mathrm{mg}$ p-Xylol $/ \mathrm{m}^{3}$ verzeichnet [46]. Für $\mathrm{m}$-Xylol wird ein $\mathrm{RD}_{50}$ von $6000 \mathrm{mg} / \mathrm{m}^{3}$, für ein Gemisch aller drei Isomere (keine näheren Angaben) ein $\mathrm{RD}_{50}$ von $10700 \mathrm{mg} / \mathrm{m}^{3}$ angegeben [47]. 


\begin{tabular}{|c|c|c|c|c|c|c|}
\hline $\begin{array}{l}\text { Spezies, Stamm, Anzahl, } \\
\text { Geschlecht pro Gruppe }\end{array}$ & Exposition & Xylol-Isomer & $\begin{array}{l}\text { NOAEC } \\
{\left[\mathrm{mg} / \mathrm{m}^{3}\right]}\end{array}$ & $\begin{array}{l}\text { LOAEC } \\
{\left[\mathrm{mg} / \mathrm{m}^{3}\right]}\end{array}$ & Untersuchter Parameter/Wirkung & Ref. \\
\hline Ratte, Wistar, $12 \mathrm{M}$ & $6 \mathrm{~h} / \mathrm{d}, 5 \mathrm{~d} / \mathrm{w}, 6 \mathrm{mo}$ & m-Xylol & - & 440 & $\begin{array}{l}\text { Verschlechterung im Rotarod-Test, Abnahme } \\
\text { der Spontanaktivität }\end{array}$ & [68] \\
\hline Ratte, Wistar, $12 \mathrm{M}$ & $6 \mathrm{~h} / \mathrm{d}, 5 \mathrm{~d} / \mathrm{w}, 3 \mathrm{mo}$ & m-Xylol & 220 & 440 & Verschlechterung im Rotarod-Test & [78] \\
\hline Ratte, Wistar, $20 \mathrm{M}$ & $6 \mathrm{~h} / \mathrm{d}, 5 \mathrm{~d} / \mathrm{w}, 3 \mathrm{mo}$ & m-Xylol & - & 440 & Verschlechterung im Verhaltenstest & [70] \\
\hline Ratte, SD, $16 \mathrm{M}$ & $6 \mathrm{~h} / \mathrm{d}, 5 \mathrm{~d} / \mathrm{w}, 13$ wo & p-Xylol & 2000 & 4000 & Ototoxizität, Cochleaschäden & [74] \\
\hline Ratte, SD, $16 \mathrm{M}$ & $6 \mathrm{~h} / \mathrm{d}, 5 \mathrm{~d} / \mathrm{w}, 13$ wo & $\begin{array}{l}\text { o-Xylol } \\
\text { m-Xylol }\end{array}$ & $\begin{array}{l}7900 \\
7900\end{array}$ & - & Ototoxizität, Cochleaschäden & [74] \\
\hline Ratte, SD, $14 \mathrm{M}$ & $6 \mathrm{~h} / \mathrm{d}, 6 \mathrm{~d} / \mathrm{w}, 13 \mathrm{wo}$ & $\begin{array}{l}\text { o-/m-Xylole, } \\
20 \% \text { p-Xylol, } \\
20 \% \text { Ethyl- } \\
\text { benzol }\end{array}$ & - & 1100 & Ototoxizität, Cochleaschäden & [75] \\
\hline Ratte, SD, 14 M & $6 \mathrm{~h} / \mathrm{d}, 6 \mathrm{~d} / \mathrm{w}, 13 \mathrm{wo}$ & $\begin{array}{l}\text { o-/m-Xylole, } \\
10 \% \text { p-Xylol, } \\
10 \% \text { Ethyl- } \\
\text { benzol }\end{array}$ & 2200 & 4400 & Ototoxizität, Cochleaschäden & [74] \\
\hline
\end{tabular}

\subsection{Neurotoxizität}

\subsubsection{Humanstudien}

Eine vierstündige Exposition von 16 männlichen Probanden gegenüber $310 \mathrm{mg} \mathrm{p}$-Xylol $/ \mathrm{m}^{3}$ hatte gleich nach Beginn sowie 2 und $4 \mathrm{~h}$ später keinen Einfluss auf Reaktionszeit und Kurzzeitgedächtnis. Eine halbstündige Exposition mit $440 \mathrm{mg} / \mathrm{m}^{3}$ führte zu einer Beeinträchtigung der Wahlreaktionszeit, der Effekt trat aber bei anderen Probanden mit höherer Belastung von 880 bzw. $1760 \mathrm{mg} / \mathrm{m}^{3}$ nicht auf [48].

In einer anderen Untersuchung traten nach einer vierstündigen Exposition von 10 männlichen Probanden mit $440 \mathrm{mg}$ Xylole $/ \mathrm{m}^{3}$ signifikant verlängerte Reaktionszeiten auf [49]. Auch nach 70-minütiger Exposition von 15 Probanden mit $100 \mathrm{ml}$ technischem Xylol/m $\mathrm{m}^{3}$ (mit 20\% Ethylbenzol) waren das Kurzzeitgedächtnis und andere zentralnervöse Funktionen beeinträchtigt [50].

$440 \mathrm{mg}$ Xylole $/ \mathrm{m}^{3}$ hatten bei $3 \mathrm{~h} \mathrm{Ex}$ position mit dreimaliger Spitzenbelastung von $880 \mathrm{mg} / \mathrm{m}^{3}$ über je $10 \mathrm{~min}$ bei sechs Probanden keinen Einfluss auf das Körpergleichgewicht. Eine Beeinträchtigung des Gleichgewichts trat aber bei der anschließenden Exposition gegenüber $880 \mathrm{mg} / \mathrm{m}^{3}$ über $3 \mathrm{~h}$ mit 10 -min-Spitzen von $1760 \mathrm{mg} / \mathrm{m}^{3}$ auf [51]. Eine vierstündige Exposition mit $640 \mathrm{mg} / \mathrm{m}^{3}$ oder $1200 \mathrm{mg} / \mathrm{m}^{3}$ hatte bei 10 Probanden keinen Einfluss auf Gleichgewichtssinn und Flimmerverschmelzungsfrequenz [52]. Dagegen wurden Gleichgewichtssinn und
Sehfähigkeit durch $4 \mathrm{~h}$ Exposition gegenüber $880 \mathrm{mg} \mathrm{m}$-Xylol/ $/ \mathrm{m}^{3}$ beeinträchtigt $[53,54]$, bei $640 \mathrm{mg} \mathrm{m-Xylol} / \mathrm{m}^{3}$ waren die Effekte auf den Gleichgewichtssinn nicht konsistent und die Sehfähigkeit nicht beeinflusst $[52,55]$.

Wurden Probanden $5 \mathrm{~h}$ gegenüber $590 \mathrm{mg} \mathrm{m}-\mathrm{Xylol} / \mathrm{m}^{3}$ mit 10 -min-Spitzen von $1760 \mathrm{mg} / \mathrm{m}^{3}$ exponiert, so war am Ende der Exposition die Reaktionszeit verlängert. Bei kontinuierlicher Belastung mit $880 \mathrm{mg} / \mathrm{m}^{3}$ wurde kein derartiger Effekt beobachtet. Nach einem Tag mit derartiger konstanter Exposition war die Zahl der Schlafbewegungen reduziert, die Schlafdauer verlängert [56].

In einigen Studien mit kontrollierter Exposition von Probanden wurden auch Befunde nach wiederholter Exposition erhoben. In einer dieser Untersuchungen wurden 9 Personen mit zwei Wochen Abstand jeweils 4 h gegenüber $880 \mathrm{mg} / \mathrm{m}^{3}$ exponiert. Dabei traten weder Gleichgewichtsstörungen noch nennenswerte Effekte auf Reaktionszeiten, Nystagmus und Flimmerverschmelzungsfrequenz auf [57]. Auch nach 9 Wochen Exposition von 10 Probanden einmal wöchentlich für jeweils $4 \mathrm{~h}$ mit $640 \mathrm{mg}$ Xylo$\mathrm{le} / \mathrm{m}^{3}$ traten keine Gleichgewichtsstörungen auf, wohl aber bei $1280 \mathrm{mg} / \mathrm{m}^{3}$ [58].

In weiteren Untersuchungen derselben Arbeitsgruppe war nach Exposition gegenüber $440 \mathrm{mg} \mathrm{m-Xylol} / \mathrm{m}^{3}$ an $6 \mathrm{~h} / \mathrm{d}$, $5 \mathrm{~d} /$ Woche am ersten, dritten und fünften Tag die Reaktionszeit im Test verlängert [59]. Nach weiterer Exposition derselben Probanden an $3 \mathrm{~d}$ in der nächsten Wo- che für jeweils $6 \mathrm{~h} / \mathrm{d}$ mit $440 \mathrm{mg}$ m-Xylol/ $\mathrm{m}^{3}$ mit jeweils sechs 10-min-Expositionsspitzen von $880 \mathrm{mg} / \mathrm{m}^{3}$ im Stundenabstand traten ebenfalls Gleichgewichtsstörungen und verlangsamte Reaktionszeiten auf [59]. Diese beiden Veränderungen traten auch am ersten, dritten und fünften Tag einer Exposition über $6 \mathrm{~h} / \mathrm{d}$ mit $400 \mathrm{mg} / \mathrm{m}^{3}$ auf, ebenso bei zusätzlicher 10-min-Spitzenexposition mit $880 \mathrm{mg} /$ $\mathrm{m}^{3}$ im Stundenabstand [60].

In einer anderen Untersuchung führte eine Exposition von 1-4 männlichen Probanden für jeweils $1 \mathrm{~h} / \mathrm{d}$ an $5 \mathrm{~d} / \mathrm{Wo}$ che für 4 Wochen mit $440 \mathrm{mg}$ p-Xylol $/ \mathrm{m}^{3}$ in der ersten Woche und jeweils 90, 660 bzw. $440 \mathrm{mg} / \mathrm{m}^{3}$ in den folgenden Wochen zu Reizeffekten bei $440 \mathrm{mg} / \mathrm{m}^{3}$, jedoch zu keinen absicherbaren Veränderungen in neurologischen Tests. Ein entsprechendes Ergebnis zeigte sich nach 5 -tägiger Exposition mit $440 \mathrm{mg} / \mathrm{m}^{3}$ bei 2-3 Frauen pro Gruppe [61]. Die geringe Gruppengröße lässt keine belastbaren Aussagen zu.

In einer Querschnittsstudie wurde aus einer Gruppe von 994 Arbeitern mit Lösemittelexposition eine Teilgruppe von 175 Arbeitern (107 Männer, 68 Frauen) untersucht, bei über $70 \%$ der Lösemittelexposition auf Xylole entfiel und bei denen alle vorgesehenen Untersuchungen durchgeführt werden konnten [62]. Als Kontrolle (vergleichbar in Alter, Geschlechterverhältnis und Raucherstatus) dienten 241 nicht exponierte Personen aus derselben und anderen Fabriken der Region. Die durchschnittliche Beschäfti- 
gungsdauer lag bei etwa 7 Jahren, in dieser Zeit hatte es keine grundlegenden Veränderungen am Arbeitsplatz gegeben. Die Exposition gegenüber Xylolen erfolgte bei der Herstellung von Plastikstiefeln und kunststoffbeschichtetem Draht sowie in der Drucktechnik. Die Höhe der Exposition wurde mittels individuellen Passivsammlern während einer 8-h-Schicht erfasst, zusätzlich wurden Urinproben am Ende der Schicht auf Methylhippursäuren untersucht. Die durchschnittliche Belastung lag in der Summe aller Xylole (Anteil o-/m-/p-Xylol ca. 15/50/30) im arithmetischen Mittel bei $92 \mathrm{mg} / \mathrm{m}^{3}$, im geometrischen Mittel (GM) bei $62 \mathrm{mg} / \mathrm{m}^{3}$, das Maximum der durchschnittlichen Exposition bei $770 \mathrm{mg} / \mathrm{m}^{3}$. Außer Xylolen wurden Toluol (GM: $14 \mathrm{mg} / \mathrm{m}^{3}$ ) und Toluol (GM: $5 \mathrm{mg} / \mathrm{m}^{3}$ ), vereinzelt auch n-Hexan, nachgewiesen; Benzol wurde bei keiner Messung gefunden. Die Höhe der Xylolbelastung der Luft korrelierte für alle drei Isomere signifikant mit der Ausscheidung der jeweiligen Methylhippursäure im Urin. Angaben zu subjektiven Symptomen während der Arbeitszeit wurden in einem Fragebogen mit 12 Fragen, Symptome während der letzten drei Monate mit 57 Fragen (Männer) bzw. 59 Fragen (Frauen) erhoben. Außerdem erfolgten Untersuchungen auf Veränderungen klinisch-chemischer und hämatologischer Parameter.

In der exponierten Gruppe wurde sowohl von Männern als auch von Frauen signifikant häufiger als in der Kontrollgruppe über Symptome während der Arbeit und in den letzten drei Monaten berichtet. Als akute Symptome wurden Augen- und Nasenreizung, Rachentrockenheit und leichtes Schwindelgefühl angegeben, als Symptome der letzten drei Monate Alpträume, Erbrechen, Angst, Vergesslichkeit, Konzentrationsschwäche, Schwindel nach schnellem Aufstehen, Appetitverlust, reduzierte Greifkraft und raue Haut angegeben. Akute Symptome, die in Zusammenhang mit einer Xyloleexposition am häufigsten genannt wurden, traten in der Tendenz bei höherer Exposition $\left(>90 \mathrm{mg} / \mathrm{m}^{3}\right)$ häufiger auf als bei geringerer $\left(4-88 \mathrm{mg} / \mathrm{m}^{3}\right)$ [62].

Eine parallel durchgeführte Untersuchung umfasste 233 Exponierte, bei denen $90 \%$ der Lösemittelanteile auf To- luol und Xylol entfielen [63]. Auch hier wurden in der exponierten Gruppe häufiger Symptome berichtet, eine Dosisabhängigkeit wurde aber ebenso wenig festgestellt.

\subsubsection{Tierexperimentelle Untersuchungen}

Die neurotoxische Wirkung von Xylolen wurde tierexperimentell in Kurzzeitstudien [64-67], in subakuten und in subchronischen Studien untersucht. Die Befunde der subchronischen Inhalationsuntersuchungen sind in $\bullet$ Tab. 3 zusammengefasst.

Auswirkungen einer subchronischen Exposition gegenüber m-Xylol auf die neuromuskuläre Koordination wurden mithilfe des Rotarods-Tests untersucht [68]. Dazu wurden je 12 männliche Wistar-Ratten pro Gruppe gegenüber 0, 440 oder $4400 \mathrm{mg} \mathrm{m}-\mathrm{Xylol} / \mathrm{m}^{3} 6$ Monate lang über 6 h/d, $5 \mathrm{~d} / \mathrm{w}$ exponiert. Vor Beginn des Versuchs wurden die Tiere trainiert, mindestens 2 min auf einem rotierenden Stab zu balancieren, und anschließend zu festgelegten Zeiten nach der Exposition untersucht. $24 \mathrm{~h}$ nach Ende der Exposition schnitten die m-Xylol-exponierten Tiere deutlich schlechter als ab die Tiere der Kontrollgruppe: Während letztere nach 3 und 6 Monaten keine Fehler im Rotarod-Test zeigten, machten $35 \%$ der Tiere mit der niedrigeren Exposition und $60 \%$ der Tiere mit der höheren Exposition Fehler. Außerdem war die Spontanaktivität der Tiere nach Exposition mit $440 \mathrm{mg} / \mathrm{m}^{3}$ im Vergleich zur Kontrollgruppe um die Hälfte vermindert (für die höhere Exposition wurden keine Daten angegeben).

In einer weiteren subchronischen Inhalationsstudie derselben Arbeitsgruppe wurden wiederum je 12 männliche Wistar-Ratten pro Gruppe (24 in der Kontrolle) an $6 \mathrm{~h} / \mathrm{d}, 5 \mathrm{~d} / \mathrm{w}$ über 3 Monate gegenüber 0,220 oder $440 \mathrm{mg} \mathrm{m}$-Xylol/ $\mathrm{m}^{3}$ exponiert und mittels Rotarod-Test auf neuromuskuläre Beeinträchtigungen hin untersucht [69]. Der Test wurde vor Beginn der Exposition und jeweils monatlich während der Studie durchgeführt (Zeitpunkt nicht genannt, jedoch vermutlich wie zuvor $24 \mathrm{~h}$ nach Expositionsende). Die Ergebnisse des RotarodTests wurden nur graphisch präsentiert.
Bei der niedrigeren Exposition gegenüber $220 \mathrm{mg} \mathrm{m}$-Xylol $/ \mathrm{m}^{3}$ wurden zu keinem Zeitpunkt signifikante Veränderungen gegenüber der Kontrolle beobachtet (Fehlerquote Kontrolle: $0 \%$, bei $220 \mathrm{mg} /$ $\mathrm{m}^{3}$ : ca. 8\%). $440 \mathrm{mg} \mathrm{m-Xylol} / \mathrm{m}^{3}$ führten nach ein, zwei und drei Monaten zu identischen signifikant erhöhten Fehlerraten von jeweils ca. $35 \%$. Leider ist in der Publikation weder beschrieben, wie oft die Tiere zu jedem Zeitpunkt bei jeder Konzentration den Rotarod-Test absolvieren mussten, noch wurden Streuungsmaße angegeben.

Einflüsse auf die Entwicklung des zentralen Nervensystems und persistierende Auswirkungen durch m-Xylol wurden in einem Verhaltenstest auf räumliche Orientierung und Gedächtnis (radial maze performance) untersucht [70]. Dazu wurden je 20 acht Wochen alte männliche Wistar-Ratten 3 Monate $(6 \mathrm{~h} / \mathrm{d}, 5 \mathrm{~d} / \mathrm{w})$ gegenüber 0, 440 oder $4400 \mathrm{mg} \mathrm{m}$-Xylol/ $\mathrm{m}^{3}$ exponiert. Untersuchungen im mazeTest wurden ab dem 70. Tag bis zum 83 . Tag der Exposition durchgeführt. Tiere der Kontrollgruppe benötigten mit zunehmender Versuchszeit und damit Übung signifikant weniger Zeit zum erfolgreichen Bewältigen des strahlenförmigen Armlabyrinths. Ein solcher Effekt trat bei den $\mathrm{m}$-Xylol-exponierten Tieren nicht auf. Außerdem machten die Tiere der Kontrollgruppe im Unterschied zu den $\mathrm{m}$-Xylol-exponierten Tieren mit zunehmender Zahl von Tagen signifikant weniger Fehler beim Erforschen des Labyrinths. Die genannten Unterschiede deuten auf eine Beeinträchtigung des Lernvermögens bei den m-Xylol-exponierten Tieren hin. In einer Anschlussuntersuchung zeigten sich nach einer 4-wöchigen Exposition gegenüber $440 \mathrm{mg} \mathrm{m}$-Xylol $/ \mathrm{m}^{3}$ keine Unterschiede zwischen Kontrolle und exponierter Gruppe [71].

Einige Alkylbenzole zeigten in Untersuchungen an Ratten ototoxische Wirkungen. Diese Wirkung kann anhand von Verhaltensänderungen, mit elektrophysiologischen Methoden oder histopathologisch anhand der Zerstörung von Haarzellen im Innenohr nachgewiesen werden. Auch mit Xylol-Isomeren oder Gemischen wurden Untersuchungen auf derartige Wirkungen durchgeführt. 
Die Ototoxizität von 21 Alkyl- und Alkenylbenzolen an männlichen SpragueDawley-Ratten wurde anhand der histologisch nachweisbaren Schädigung der Haarzellen im Innenohr verglichen [72]. Nach subakuter oraler Verabreichung ( $2 \mathrm{ml} / \mathrm{kg}$ und Tag in Öl per Schlundsonde, $5 \mathrm{~d} / \mathrm{w}, 2$ Wochen) erwies sich von den Xylolen nur p-Xylol als ototoxisch, jedoch geringer ototoxisch als Ethylbenzol. Diese Unterschiede in der Ototoxizität konnten in einer subakuten Inhalationsstudie bestätigt werden, in der nach dreiwöchiger Exposition gegenüber jeweils $7900 \mathrm{mg} / \mathrm{m}^{3}$ nur p-Xylol zu Cochleaschäden im Innenohr führte [73].

In einer subchronischen Inhalationsstudie [74] wurden auditorisch evozierte Potentiale im Hirnstamm zur Bestimmung der Hörschwelle bei unterschiedlichen Frequenzen bei männlichen Sprague-Dawley-Ratten (16/Gruppe) 8 Wochen nach Ende einer 13wöchingen Exposition $(6 \mathrm{~h} / \mathrm{d}, 6 \mathrm{~d} /$ Woche $)$ gegenüber 2000, 4000 oder $7900 \mathrm{mg} / \mathrm{m}^{3}$ des jeweiligen Xylol-Isomers untersucht. Nur p-Xylol wirkte ab $3900 \mathrm{mg} / \mathrm{m}^{3}$ mäßig bis schwer ototoxisch mit erhöhter Hörschwelle und mäßigem bis starkem Verlust von Hörzellen im Cortischen Organ.

In einer entsprechenden subchronischen Inhalationsstudie mit zwei technischen Xylole-Gemischen (Mischung 1: je $20 \%$ o- und p-Xylol sowie Ethylbenzol, $40 \%$ m-Xylol; Mischung 2: 30\% o-, je $10 \%$ p-Xylol und Ethylbenzol, $50 \%$ m-Xylol) zeigte sich in Übereinstimmung mit der höheren Ototoxizität von p-Xylol und Ethylbenzol eine niedrigere LOAEC für das Gemisch mit dem höheren Gehalt an diesen beiden Komponenten [75]. Damit wurden vorangegangene subakute Untersuchungen mit Xylole-Gemischen bestätigt $[76,77]$.

Untersuchungen $\mathrm{zu}$ neurotoxischen Effekten nach chronischer inhalativer Exposition liegen nicht vor.

\subsection{Gentoxizität und Kanzerogenität}

Zahlreiche Mutagenitätsstudien an Bakterien (Escherichia coli und Salmonella typhimurium) mit technischen $\mathrm{Xy}$ lol oder einzelnen Xylol-Isomeren ergaben in Ab- und Anwesenheit von exo- genem metabolischen Aktivierungssystem durchweg keine Hinweise auf gentoxische Wirkungen. Auch in einem Invitro-Test mit Mauslymphomzellen wurde keine mutagene Wirkung festgestellt. Ebenso wurden in Ovarzellen des Chinesischen Hamsters oder in Humanlymphozyten (nur in Abwesenheit exogenen metabolischen Systems getestet) in vitro nach Behandeln mit technischen Xylol weder vermehrt Schwesterchromatidaustausche noch Chromosomenaberrationen beobachtet $[1,3]$. In einer Untersuchung („comet assay“) an Humanlymphozyten in vitro fanden sich bei einer Konzentration von $200 \mu \mathrm{mol} / \mathrm{l}(21 \mathrm{mg} / \mathrm{l}) \mathrm{Hin}$ weise auf eine DNA-schädigende Wirkung (Doppelstrangbrüche), möglicherweise infolge der Bildung freier Radikale, die zu einer oxidativen Veränderung von DNA-Basen führen [79]. Hingegen wurde in Untersuchungen an Lymphozyten von Beschäftigten mit beruflicher oder experimenteller Xylolexposition kein vermehrtes Auftreten von Schwesterchromatidaustauschen beobachtet [80]. Technisches Xylol, nicht aber o- und m-Xylol, zeigten im SLRL-Test an der Fruchtfliege Drosophila melanogaster schwache mutagene Effekte. Untersuchungen auf die Induktion von Mikrokernen im Knochenmark von Mäusen in vivo erbrachten weder für Einzelisomere noch für technisches Xylol Hinweise auf klastogene Effekte (Chromosomenaberrationen und Mikronuklei) $[1,3]$. In der Gesamtbewertung lassen die Befunde nicht auf ein gentoxisches Potential der Xylole schließen.

Belastbare Hinweise auf eine kanzerogene Wirkung von Xylolen liegen aus epidemiologischen Studien nicht vor [42].

Bei CFY-Ratten zeigten sich nach bis zu einjähriger Exposition gegenüber $4750 \mathrm{mg}$ o-Xylol/m³ über $8 \mathrm{~h} / \mathrm{d}, 7 \mathrm{~d} / \mathrm{Wo}$ che außer einer verminderten Gewichtszunahme eine Proliferation des endoplasmatischen Retikulums in der Leber ohne adverse histopathologische oder klinischchemische Veränderungen [81].

In einer Untersuchung an SpragueDawley-Ratten war nach Verabreichen von $500 \mathrm{mg}$ Xylole/kg KG und Tag (keine Angaben zur Reinheit) per Schlundsonde an 4-5 d/Woche über insgesamt zwei Jahre die Gesamtzahl maligner Tumoren gegenüber der Kontrollgruppe numerisch erhöht (Kontrolle: 12/50 M, 11/50 W, behandelt: 18/40 M, 26/40 W) [82]. Eine statistische Analyse und systematische Darstellung aller Tumoren wurde nicht vorgenommen.

In einer NTP-Studie wurde F344-Ratten 103 Wochen 0, 250 oder $500 \mathrm{mg} / \mathrm{kg}$ KG und Tag technisches Xylol (9\% o-, $60 \%$ m-, $14 \%$ p-Xylol sowie $17 \%$ Ethylbenzol) in Maisöl per Schlundsonde verabreicht; B6C3F1-Mäuse erhielten 0, 500, 1000 mg/kg KG und Tag. In keiner der beiden Studien fanden sich Hinweise auf eine kanzerogene Wirkung [83].

\subsection{Reproduktionstoxizität}

Bewertungsrelevante Daten beim Menschen liegen nicht vor. Untersuchungen an Ratten, Mäusen oder Kaninchen lieferten keine klaren Hinweise auf Beeinträchtigungen der Fortpflanzungsfähigkeit oder spezifische teratogene Wirkungen von Xylolen [84-88]. Auch hinsichtlich entwicklungsneurotoxischer Wirkungen ist die Evidenz schwach [89-94].

\subsection{Geruchswahrnehmung}

Die Bewertung der Wahrnehmung von Gerüchen orientiert sich, wenn möglich, an der Wahrnehmungsschwelle. Diese stellt konventionsgemäß diejenige Konzentration dar, bei der die Hälfte der angebotenen Geruchsproben von dem Untersuchungskollektiv wahrgenommen wird.

Als Geruchswahrnehmungsschwelle wurde für o-Xylol $1,7 \mathrm{mg} / \mathrm{m}^{3}$, für $\mathrm{m}-\mathrm{Xy}$ lol 0,18 mg/m $\mathrm{m}^{3}$ und für $\mathrm{p}$-Xylol 0,26 mg/ $\mathrm{m}^{3}$ ermittelt [95].

\subsection{Kombinationswirkung mit anderen Stoffen}

Hinweise auf überadditive Wirkungen von Xylolen und Toluol wurden im Rotarod-Test an Ratten beschrieben, bei dem stärkere Veränderungen durch jeweils $50 \mathrm{ml}$ Toluol und Xylole $/ \mathrm{m}^{3}$ als durch $220 \mathrm{mg} \mathrm{m}-\mathrm{Xylol} / \mathrm{m}^{3}$ allein auftraten [68]. Dagegen führte technisches Xylol (32\% p-Xylol, 2,5\% Ethylbenzol) bei $4400 \mathrm{mg} /$ $\mathrm{m}^{3}$ bei Ratten zu einer weniger als additiven Verstärkung des durch n-Hexan verursachten Hörverlustes [77]. Bei kontrollierter Exposition von Probanden wurden 


\begin{tabular}{|c|c|c|c|c|c|c|c|c|c|}
\hline \multirow[t]{2}{*}{ Endpunkt } & \multirow{2}{*}{$\begin{array}{l}\text { LOAEC/NOAEC } \\
\left(\mathrm{mg} / \mathrm{m}^{3}\right)^{\mathrm{a}}\end{array}$} & \multicolumn{5}{|l|}{ Faktor } & \multirow{2}{*}{$\begin{array}{l}\text { Leitwert } \\
\left(\mathrm{mg} / \mathrm{m}^{3}\right)\end{array}$} & \multirow{2}{*}{$\begin{array}{l}\text { Organisation } \\
\text { (Jahr) }\end{array}$} & \multirow[t]{2}{*}{ Ref. } \\
\hline & & $\begin{array}{l}\text { LOAEC zu } \\
\text { NOAEC }\end{array}$ & Interspezies & Intra-spezies & Zeit & Datenlage & & & \\
\hline $\begin{array}{l}\text { Neurotoxizität, Irritation, } \\
\text { Mensch }\end{array}$ & 62 (LOAEC) & - & 10 & 10 & - & 3 & 0,2 & $\begin{array}{l}\text { US-ATSDR } \\
\text { (2007) }\end{array}$ & [31] \\
\hline Neurotoxizität, Ratte & 220 (NOAEC) & - & 3 & 10 & 3 & 3 & 0,1 & US-EPA (2003) & $\begin{array}{l}{[1,} \\
99]\end{array}$ \\
\hline Fetotoxizität, Ratte & 350 (LOEC) & 10 & 10 & 10 & - & - & 0,18 & $\begin{array}{l}\text { Health Canada } \\
\text { (1993) }\end{array}$ & [24] \\
\hline $\begin{array}{l}\text { Neurotoxizität, Irritation, } \\
\text { Mensch }\end{array}$ & 62 (LOAEC) & 3 & - & 10 & - & - & 0,7 & $\begin{array}{l}\text { Cal-OEHHA } \\
(2000)\end{array}$ & [25] \\
\hline $\begin{array}{l}\text { Entwicklungsneurotoxi- } \\
\text { zität, Ratte }\end{array}$ & 870 (LOAEC) & 10 & 10 & 10 & - & - & 0,87 & $\begin{array}{l}\text { RIVM-NL } \\
\text { (1991) }\end{array}$ & [98] \\
\hline $\begin{array}{l}\text { Entwicklungsneurotoxi- } \\
\text { zität, Ratte }\end{array}$ & 870 (LOAEC) & 10 & 10 & 10 & - & - & 0,87 & WHO (1997) & [9] \\
\hline
\end{tabular}

\begin{tabular}{|c|c|c|c|c|c|c|c|c|}
\hline \multirow[t]{2}{*}{ Endpunkt } & \multirow{2}{*}{$\begin{array}{l}\text { LOAEC/NOAEC } \\
\left(\mathrm{mg} / \mathrm{m}^{3}\right)\end{array}$} & \multicolumn{5}{|c|}{ Extrapolationsfaktor } & \multirow[t]{2}{*}{ Wert $\left(\mathrm{mg} / \mathrm{m}^{3}\right)$} & \multirow[t]{2}{*}{ Basisstudie } \\
\hline & & $\begin{array}{l}\text { LOAEC zu } \\
\text { NOAEC }\end{array}$ & Interspezies & Intra-spezies & Zeit & „Kinderfaktor" & & \\
\hline Neurotoxizität, Ratte & $440\left(78,6^{\mathrm{a}}\right)(\mathrm{LOAEC})$ & - & 2,5 & 10 & 2 & 2 & 0,79 & {$[68,69]$} \\
\hline $\begin{array}{l}\text { Neurotoxizität, Irrita- } \\
\text { tion, Mensch }\end{array}$ & $\begin{array}{l}62\left(14,8^{\mathrm{a}}\right) \text { (minimal } \\
\text { LOAEC) }\end{array}$ & - & - & 10 & 1 & 2 & 0,73 & [62] \\
\hline Ototoxizität, Ratte & $1100\left(196^{\mathrm{a}}\right)(\mathrm{LOAEC})$ & - & 2,5 & 10 & 2 & 2 & 1,96 & [75] \\
\hline
\end{tabular}

Effekte von Xylol auf den Gleichgewichtssinn durch Ethanol verstärkt [42]. In anderen Untersuchungen wurden ab $50 \mathrm{ml}$ Toluol und Xylol/ $\mathrm{m}^{3}$ kompetitive Hemmungen im Metabolismus beider Stoffe festgestellt [42]. Butanon hemmt die Seitenkettenoxidation von $\mathrm{m}$-Xylol und mindert daher beim Menschen die Bildung und Ausscheidung von Methylhippursäure im Urin bei gleichzeitigem Anstieg des Xylol-Gehalts in Blut und Fettgewebe [31].

\section{Bewertung}

Die wesentlichen toxischen Wirkungen von Xylolen, die bei niedrigen Expositionskonzentrationen beim Menschen und tierexperimentell belegt sind, bestehen in Beeinträchtigungen des Nervensystems. Daneben wurden in diesem Konzentrationsbereich beim Menschen auch Reizeffekte an Augen und Schleimhäuten des oberen Atemtrakts beschrieben.

Nach einmaliger oder wiederholter Kurzzeitexposition zeigten sich bei Probanden erste Wirkungen auf das zentra- le Nervensystem bei Konzentrationen um $440 \mathrm{mg}$ Xylole $/ \mathrm{m}^{3}$. In der einzigen bewertbaren Langzeitstudie [62] gaben die Beschäftigten nach Exposition gegenüber Xylolen signifikant häufiger als in der Kontrollgruppe akute Symptome wie Augen- und Nasenreizung und leichtes Schwindelgefühl sowie in den Monaten vor der Befragung häufiger zahlreiche gesundheitliche Beeinträchtigungen an. Die durchschnittliche Beschäftigungsdauer lag bei 7 Jahren, die Exposition zum Zeitpunkt der Untersuchung im geometrischen Mittel bei $62 \mathrm{mg} / \mathrm{m}^{3}$, die maximale durchschnittliche Exposition erreichte $770 \mathrm{mg} / \mathrm{m}^{3}$. Angaben über Häufigkeit, Dauer und Höhe von Belastungsspitzen fehlen ebenso wie Angaben zur Höhe der Belastung in den vergangenen Jahren. Insgesamt werden die Humanstudien nicht als ausreichend belastbar für eine Ableitung von Richtwerten angesehen.

Bei Ratten wurden neurologische Effekte in Form von Beeinträchtigungen der neuromuskulären Koordination, des Gedächtnisses und des Hörvermögens beschrieben. Ototoxische Effekte zeigte nur das p-Xylol, nicht aber o- oder m-Xylol. Bei anderen neurologischen Endpunkten sprechen die Befunde dafür, dass keine bewertungsrelevanten Unterschiede in der Toxizität der Xylol-Isomere vorhanden sind. Die niedrigste Konzentration, bei der noch adverse neurologische Effekte auftraten, wurden mit $440 \mathrm{mg} \mathrm{Xy-}$ lole $/ \mathrm{m}^{3}$ in zwei subchronischen Inhalationsstudien an Ratten berichtet $[68,69]$, in denen Beeinträchtigungen der neuromuskulären Koordination beschrieben wurden, sowie in einer weiteren Studie, in der bei Ratten Hinweise auf ein beeinträchtigtes Lernvermögen beschrieben wurden [70].

Die vorliegenden Ergebnisse aus Studien zur Entwicklungstoxizität liefern keine belastbaren Hinweise auf eine Beeinträchtigung der neurologischen Entwicklung von Jungtieren trächtiger Xylol-exponierter Weibchen. Aus Studien zur Entwicklungstoxizität von o-Xylol ergab sich eine NOAEC von $440 \mathrm{mg} / \mathrm{m}^{3}$ für fötotoxische Effekte (vermindertes Körpergewicht der Föten) [89, 90]. 


\subsection{Bestehende Regelungen}

Weder im Gefahrstoffrecht der EU noch von der Internationalen Krebsforschungsbehörde liegen für Xylole Einstufungen hinsichtlich einer kanzerogenen Wirkung vor.

Vom europäischen Wissenschaftlichen Ausschuss für Expositionsbegrenzung am Arbeitsplatz wurde 1992 ein Wert von $221 \mathrm{mg}$ Xylole $/ \mathrm{m}^{3}$ vorgeschlagen [5]. Der Ausschuss stützte sich in der Begründung dieses Werts auf die in Kurzzeitstudien beim Menschen beobachteten Reizwirkungen auf Augen und den oberen Atemtrakt sowie auf zentralnervöse Effekte, die bei Konzentrationen um $440 \mathrm{mg}$ Xylole $/ \mathrm{m}^{3}$ auftraten.

Die Arbeitsstoff-Kommission der Deutschen Forschungsgemeinschaft sah die Befunde der Querschnittstudie [62] wegen der Nicht-Berücksichtigung von Confoundern als nicht geeignet für die Bewertung an. Sie hatte bereits 1983 auf der Basis von Humanstudien mit Kurzzeitexposition gegenüber Xylolen, bei der im Bereich von $400-1200 \mathrm{mg} / \mathrm{m}^{3}$ Hinweise auf Beeinträchtigungen des Gleichgewichtsorgans beschrieben wurden, eine Maximale Arbeitsplatzkonzentration von $440 \mathrm{mg}$ Xylole $/ \mathrm{m}^{3}$ festgelegt [4], der 1998 [42] bestätigt und in dieser Höhe in die TRGS 900 übernommen wurde [96].

Vom Ausschuss für Gefahrstoffe wurde 2008 ein Arbeitsplatzgrenzwert (AGW) von $200 \mathrm{mg} / \mathrm{m}^{3}$ für $\mathrm{C}_{7}-\mathrm{C}_{8}$-Aromaten festgelegt, der bei der Bewertung von Kohlenwasserstoffgemischen am Arbeitsplatz gemäß der RCP-Methode (reciprocal calculation procedure) zu berücksichtigen ist. Sofern ein Xylol als Einzelkohlenwasserstoff einem Kohlenwasserstoffgemisch zugesetzt wird, geht der RCP-Gruppengrenzwert und nicht der stoffspezifische AGW in die Berechnung ein [97].

Bestehende Leitwerte für Xylole in der Luft zum Schutz der Allgemeinbevölkerung sind in - Tab. 4 zusammengefasst. Die kanadische Gesundheitsbehörde hatte bereits 1993 eine vorläufige tolerable Konzentration für Xylole von $0,18 \mathrm{mg} /$ $\mathrm{m}^{3}$ abgeleitet [24], basierend auf einer LOEC von $350 \mathrm{mg}$ Xylole/ $\mathrm{m}^{3}$ für fetotoxische Effekte bei Ratten (verzögerte Skelettentwicklung) [87] unter Berücksichtigung von Körpergewicht und Atemrate sowie eines Gesamtextrapolationsfaktors von 1000 (je 10 für Inter- und Intraspeziesvariabilität und zur Extrapolation auf eine NOAEC).

Von der Weltgesundheitsorganisation [9] wurde 1997 auf Basis entwicklungsneurotoxischer Effekte an Ratten mit einer LOAEC von $870 \mathrm{mg} / \mathrm{m}^{3}$ [93] und einem Gesamtextrapolationsfaktor von 1000 (je 10 zur Extrapolation auf eine NOAEC sowie für Inter- und Intraspeziesextrapolation) ein Leitwert von $0,87 \mathrm{mg}$ Xylole $/ \mathrm{m}^{3}$ abgeleitet. Einen identischen Wert auf $\mathrm{Ba}$ sis derselben Studienbefunde hat auch das niederländische Reichsinstitut für Volksgesundheit und Milieuhygiene abgeleitet [98]. $\mathrm{Zu}$ einem geringfügig niedrigeren "chronischen Referenzwert" von 0,7 mg Xylole/ $\mathrm{m}^{3}$ kam 2000 die kalifornische Behörde für die Abschätzung umweltbedingter Gesundheitsgefahren [25], deren Ableitung auf der Querschnittsstudie von Uchida et al. [62] beruht.

Die US-amerikanische Umweltbehörde hatte 2003 eine "Referenzkonzentration" (RfC) von $0,1 \mathrm{mg}$ Xylole/ $\mathrm{m}^{3}$ abgeleitet $[1,99]$. Die Festlegung basierte auf den Befunden der Untersuchungen von Korsak et al. $[68,69]$, nach denen bei subchronischer inhalativer Exposition von Ratten Wirkungen auf die neuromotorische Koordination im Rotarod-Test beobachtet wurden. Ausgehend von einer NOAEC von $220 \mathrm{mg}$ Xylole $/ \mathrm{m}^{3}$ ergab sich nach Umrechnung auf kontinuierliche Exposition mit einem Interspeziesfaktor von 3 (Toxikokinetik: 1, -dynamik: 3), einem Intraspeziesfaktor von 10, einem Faktor 3 zur Extrapolation von subchronischer auf chronische Exposition und einem Faktor von 3 wegen Unsicherheiten in der Gesamtdatenbasis (u. a. wegen Fehlens einer 2-Generationen-Studie zur Reproduktionstoxizität) die genannte RfC. Die vorliegenden Humandaten, darunter die Studie von Uchida et al. [62], wurden als unzureichend für die Ableitung einer RfC angesehen.

Im Unterschied zur Umweltbehörde sah die US-amerikanische Behörde für Gefahrstoffe und Krankheitserfassung 2007 die Querschnittstudie von Uchida et al. [62] als ausreichend valide an und leitete auf Basis dieser Studie eine „chronische minimale Risikokonzentration"von $0,2 \mathrm{mg}$ Xylole $/ \mathrm{m}^{3} \mathrm{ab}$. Ausgangspunkt wa- ren dabei die in der Studie berichteten neurologischen Wirkungen und Reizeffekte auf die Schleimhäute bei Beschäftigten bei einer chronischen Exposition gegenüber $62 \mathrm{mg}$ Xylole $/ \mathrm{m}^{3}$ (geometrisches Mittel). Die ATSDR zog bei der Ableitung einen Inter- und Intraspeziesfaktor von je 10 heran und zusätzlich einen Faktor 3 wegen des Fehlens weiterer chronischer Studien zur Neurotoxizität [31].

Für die chronische Exposition mit Xylolen im Innenraum von Kraftfahrzeugen wurde ein sog. ELIA (Exposure Level Inside Automotic Vehicles) von $8,8 \mathrm{mg} / \mathrm{m}^{3}$ vorgeschlagen [100]. Dieser Wert wurde mithilfe einer Pfad-zu-Pfad-Übertragung aus dem NOAEL der oralen chronischen NTP-Studie an Ratten [83] mit einem Gesamtextrapolationsfaktor von 100 abgeleitet.

\subsection{Ableitung von Richtwerten für Xylole in der Innenraumluft}

\subsubsection{Richtwert II}

Für die Festsetzung des Richtwertes II ist nach dem fortgeschriebenen Basisschema [101] von der niedrigsten beobachteten adversen Wirkungskonzentration (LOAEC) auszugehen.

Als kritischen Effekt sieht der Ausschuss für Innenraumrichtwerte die in subchronischen Inhalationsstudien an Ratten beobachteten neurotoxischen Wirkungen von Xylol $[68,69]$. Ein auf Basis dieser Befunde abgeleiteter Richtwert wird gestützt durch Befunde zu Wirkungen beim Menschen nach Exposition gegenüber Xylolen am Arbeitsplatz [62], auf deren Basis sich ein praktisch identischer Richtwert ergäbe (• Tab. 5). Die Humanbefunde werden für sich genommen vom Ausschuss als zu wenig abgesichert bewertet, um als Basisstudie zur Ableitung des Richtwerts herangezogen zu werden. Die im Tierversuch [75] beobachtete Ototoxizität eines Xylolgemisches mit $20 \%$ p-Xylol (wie es für technische Gemische als typisch anzusehen ist, siehe Abschnitt 1.2) als Basis der Ableitung würde demgegenüber zu einem höheren Richtwert führen.

Die niedrigste Konzentration, bei der eine signifikante Beeinträchtigung der neuromuskulären Koordination bei Ratten beschrieben wurde, lag bei $440 \mathrm{mg} \mathrm{m}$ - 
$\mathrm{Xylol} / \mathrm{m}^{3}[68,69]$. Eine Benchmarkmodellierung der Daten ist wegen des Fehlens entsprechender Angaben nicht möglich.

Bei der Extrapolation der ermittelten LOAEC auf eine lebenslange Exposition auf der Grundlage des Basisschemas werden folgende Faktoren verwendet:

- Zeitanpassung auf eine kontinuierliche Exposition: Faktoren 24/6 und $7 / 5$

- Extrapolation subchronisch $\rightarrow$ chronisch: Faktor 2

- Interspezies Toxikodynamik: Faktor 2,5

- Intraspezies: Faktor 10

- Erhöhte Atemrate bei Kindern (bezogen auf das Körpergewicht): Faktor 2

Der Gesamtextrapolationsfaktor beträgt damit 560. Daraus ergibt sich eine Konzentration von 440: $560=0,79 \mathrm{mg} / \mathrm{m}^{3}$.

Der Ausschuss legt als Richtwert II 0,8 mg Summe Xylole $/ \mathrm{m}^{3}$ fest.

\subsubsection{Richtwert I}

Nach dem Basisschema wird der Richtwert I um eine Größenordnung niedriger festgesetzt und liegt damit bei $0,1 \mathrm{mg}$ Summe Xylole $/ \mathrm{m}^{3}$.

\section{Anmerkungen}

Der Textentwurf dieser Empfehlung wurde von Dr. Jens-Uwe Voss erstellt und vom Ausschuss für Innenraumrichtwerte im Juli 2015 verabschiedet. Die Literaturrecherche wurde im September 2014 abgeschlossen.

\section{Literatur}

1. U.S.EPA (2003) Toxicological Review of Xylenes. EPA/635/R-03/001. U.S. Environmental Protection Agency, Washington, D.C. http://www.epa. gov/iris/toxreviews/0270-tr.pdf

2. ECHA (2014) Classification and Labelling Inventory: Harmonised Classification - Annex VI of Regulation (EC) No. 1272/2008 (CLP Regulation). European Chemicals Agency (ECHA). http://clp-inventory.echa.europa.eu/

3. IARC (1999) Re-evaluation of some organic chemicals, hydrazine and hydrogen peroxide. IARC Monographs on the evaluation of carcinogenic risks to humans 71. IARC (International Agency for Research on Cancer), WHO (World Health Organization), Lyon
4. DFG (1983) Xylol (alle Isomeren). Gesundheitsschädliche Arbeitsstoffe. Toxikologisch-arbeitsmedizinische Begründungen von MAK-Werten, 9. Lieferung. Wiley-VCH, Weinheim, S 1-20

5. SCOEL (1992) Recommendation from the Scientific Committee on Occupational Exposure Limits for Xylenes. SCOEL/SUM/1. European Commission, Employment, Social Affairs \& Inclusion, Health and Safety at Work. The Scientific Committee on Occupational Exposure Limits (SCOEL)

6. Hohenblum P, Fröhlich M, Moosmann L et al (2008) LUKI - LUft und KInder: Einfluss der Innenraumluft auf die Gesundheit von Kindern in Ganztagsschulen. Umweltbundesamt $\mathrm{GmbH}$, Wien

7. Hemfler M, Jacobi S, Schwarzloh D et al (2014) Lufthygienischer Jahresbericht 2014, Teil I: Kontinuierliche Messungen. Hessisches Landesamt für Umwelt und Geologie (HLUG) (Ed.), Wiesbaden. www.hlug.de

8. LfU (2014) Lufthygienischer Jahresbericht 2013. Bayerisches Landesamt für Umwelt, Referat 24 (Ed.). Augsburg. www.lfu.bayern.de

9. WHO (1997) Xylenes. Environmental Health Criteria (EHC) 190. International Programme on Chemical Safety (IPCS). World Health Organization, Geneva

10. Eis D, Helm D, Laußmann D et al (2005) Berliner Studie zu umweltbezogenen Erkrankungen. Im Auftrag des Bundesgesundheitsministeriums, Berlin. www.apug.de/archiv/pdf/Berichtsband_ Berliner-Studie.pdf

11. Moriske H-J (2002) Chemische Innenraumluftverunreinigungen. In: Moriske H.-J, Turowski E (Hrsg) Handbuch für Bioklima und Lufthygiene 8. Erg. Lfg. Chapter III-4.2. Ecomed verlagsgesellschaft, Landsberg am Lech, S 1-64

12. Schulz Ch, Ullrich D, Pick-Fuß H et al (2010) Kinder-Umwelt-Survey (KUS) 2003/06. Innenraumluft - Flüchtige organische Verbindungen in der Innenraumluft in Haushalten mit Kindern in Deutschland. Schriftenreihe Umwelt \& Gesundheit. Umweltbundesamt, Dessau-Roßlau. http:// www.uba.de/uba-info-medien/4011.html

13. Schlechter N, Pohl K, Barig A et al (2004) Beurteilung der Raumluftqualität an Büroarbeitsplätzen. Gefahrstoffe Reinhaltung der Luft 03/2004:95-99

14. von Hahn N, van Gelder R, Breuer D et al (2011) Ableitung von Innenraumarbeitsplatz-Referenzwerten. Gefahrstoffe Reinhaltung der Luft 71:314-322

15. Hofmann H Plieninger $P$ (2008) Bereitstellung einer Datenbank zum Vorkommen von flüchtigen organischen Verbindungen in der Raumluft Forschungsbericht 20561 243. Arbeitsgemeinschaft ökologischer Forschungsinstitute (AGÖF) e. V., im Auftrag des Umweltbundesamts. www. umweltdaten.de/publikationen/fpdf-1/3637.pdf

16. AGÖF (2011) Auswertung der AGÖF-Datenbank VOC-Datenbank-DB-08-2011. Detaillierte Auswertung der im Rahmen des Forschungsprojektes FKZ 20561243 erhobenen Daten für Alkylbenzole sowie Glykolester und Glykolether. Arbeitsgemeinschaft ökologischer Forschungsinstitute (AGÖF) e. V. Springe-Eldagsen, Germany

17. Hofmann H, Erdmann G, Müller A (2014) Zielkonflikt energieffiziente Bauweise und gute Raumluftqualität - Datenerhebung für flüchtige organische Verbindungen in der Innenraumluft von Wohn- und Bürogebäuden (Lösungswege). Förderkennzeichen (UFOPLAN) 370962211. Arbeitsgemeinschaft ökologischer Forschungsinstitute (AGÖF) e. V., im Auftrag des Umweltbundesamts, Dessau-Roßlau, Germany
18. Fromme H, Heitmann D, Dietrich S et al (2008) Raumluftqualität in Schulen - Belastung von Klassenräumen mit Kohlendioxid (CO2), flüchtigen organischen Verbindungen (VOC), Aldehyden, Endotoxinen und Katzenallergenen. Gesundheitswesen 70:88-97

19. Lahrz T, Piloty M, Oddoy A et al (2003) Gesundheitlich bedenkliche Substanzen in öffentlichen Einrichtungen in Berlin. Untersuchungen zur Innenraumluftqualität in Berliner Schulen. Bericht des Institus für Lebensmittel, Arzneimittel und Tierseuchen, Fachbereich Umwelt- und Gesundheitsschutz, Berlin. Zitiert nach Fromme et al. (2008)

20. Ostendorp G, Riemer D, Harmel K et al (2009) Aktuelle Hintergrundwerte zur VOC-Belastung in Schulen und Kindergärten in Schleswig-Holstein. Umweltmed. Forsch. Prax 14:135-152

21. Neumann H-D, Buxtrup M, Benitez S et al (2013) Gesunde Luft in Schulen: VOC- und Aldehydkonzentrationen in beschwerdefreien Klassenräumen. Unfallkasse Nordrhein-Westfalen, Düsseldorf, S 63

22. Ad-hoc-Arbeitsgruppe Innenraumrichtwerte der IRK/AGLMB (2012) Richtwerte für Ethylbenzol in der Innenraumluft. Bundesgesundheitsblatt 55:1192-1200

23. Ad-hoc-Arbeitsgruppe Innenraumrichtwerte der IRK/AGLMB (2012) Richtwerte für C9-C15-Alkylbenzole in der Innenraumluft. Bundesgesundheitsblatt 55:1201-1214

24. Health Canada (1993) Priority Substances List Assessment Report. Xylenes. Canadian Environmental Protection Act, Government of Canada. Environment Canada, Health, Ottawa. http:// www.hc-sc.gc.ca/ewh-semt/alt_formats/hecssesc/pdf/pubs/contaminants/psl1-Isp1/xylenes/ xylene-eng.pdf

25. OEHHA (2000) Chronic toxicity summary. Xylene. Determination of Noncancer Chronic Reference Exposure Levels. Office of Environmental Health Hazard Assessment (OEHHA), California Environmental Protection Agency, State of California, http://oehha.ca.gov/air/chronic_rels/pdf/xylensREL.pdf

26. Sedivec V, Flek J (1976) The absorption, metabolism, and excretion of xylenes in man. Int Arch Occup Environ Health 37:205-217

27. Riihimaki V, Savolainen K (1980) Human exposure to $m$-xylene. Kinetics and acute effects on the central nervous system. Ann Occup Hyg 23:411422

28. David A, Flek J, Frantik E et al (1979) Influence of phenobarbital on xylene metabolism in man and rats. Int Arch Occup Environ Health 44:117-125

29. Astrand I, Engstrom J, Ovrum P (1978) Exposure to xylene and ethylbenzene. I. Uptake, distribution and elimination in man. Scand J Work Environ Health 4:185-194

30. Senczuk W, Orlowski J (1978) Absorption of mxylene vapours through the respiratory tract and excretion of $\mathrm{m}$-methylhippuric acid in urine. $\mathrm{Br} J$ Ind Med 35:50-55

31. ATSDR (2007) Toxicological Profile for Xylenes. U.S. Department of Health and Human Services. Public Health Service. Agency for Toxic Substances and Disease Registry, Atlanta. http://www. atsdr.cdc.gov/toxprofiles/tp71.pdf

32. Kumarathasan P, Otson R, Chu I (1998) Application of an automated HS-GC method in partition coefficient determination for xylenes and ethylbenzene in rat tissues. Chemosphere 37:159-178 
33. Kumarathasan P, Otson R, Chu I (1997) Measurement of the distribution of $\mathrm{m}$-xylene in rat tissues by head space gas chromatography. Arh Hig Rada Toksikol 48:373-382

34. Carlsson A (1981) Distribution and elimination of $14 \mathrm{C}$-xylene in rat. Scand J Work Environ Health 7:51-55

35. Bergman K (1983) Application and results of whole-body autoradiography in distribution studies of organic solvents. Crit Rev Toxicol 12:59118

36. Bergman K (1979) Whole-body autoradiography and allied tracer techniques in distribution and elimination studies of some organic solvents: benzene, toluene, xylene, styrene, methylene chloride, chloroform, carbon tetrachloride and trichloroethylene. Scand J Work Environ Health 5:1-263

37. Ernstgard L, Sjogren B, Warholm M et al (2003) Sex differences in the toxicokinetics of inhaled solvent vapors in humans 1. m-Xylene. Toxicol Appl Pharmacol 193:147-157

38. Adams JC, Dills RL, Morgan MS et al (2005) A physiologically based toxicokinetic model of inhalation exposure to xylenes in Caucasian men. Regul Toxicol Pharmacol 43:203-214

39. DFG (2010) Xylenes (all isomers), Addendum. In: Deutsche Forschungsgemeinschaft (DFG) (Hrsg) The MAK-collection part IV: BAT value documentations 5. Wiley-VCH, Weinheim, S 1-8

40. NRC (2000) Spacecraft Maximum Allowable Concentrations for Selected Airborne Chemicals. 9. National Academy of Science. National Academy Press, Washington, D.C

41. NRC (2008) Spacecraft Maximum Allowable Concentrations for Selected Airborne Chemicals. Xylene. Spacecraft Maximum Allowable Concentrations (SMAC) for Selected Airborne Chemicals 5, National Academy of Science, National Academy Press, Washington, D.C, S 356-386

42. DFG (1998) Xylol (alle Isomeren). Gesundheitsschädliche Arbeitsstoffe. Toxikologisch-arbeitsmedizinische Begründungen von MAK-Werten, 27. Lieferung. Wiley-VCH, Weinheim, S 1-30

43. OEHHA (2008) TSD for Noncancer RELs. Appendix D. Individual Acute, 8-Hour, and Chronic Reference Exposure Level Summaries. Office of Environmental Health Hazard Assessment (OEHHA), California Environmental Protection Agency, State of California., http://oehha.ca.gov/air/hot_ spots/2008/AppendixD1_final.pdf

44. Hastings, L, GP Cooper, W Burg (1984) Human sensory response to selected petroleum hydrocarbons. Advances in Modern Environmental Toxicology VI. Applied Toxicology of Petroleum Hydrocarbons. Princeton Scientific Publishers, Inc., Princeton, N.J, S 255-270, Zitiert nach OEHHA (2008)

45. Ernstgard L, Gullstrand E, Lof A et al (2002) Are women more sensitive than men to 2-propanol and m-xylene vapours? Occup Environ Med 59:759-767

46. Bos P, Zwart A, Reuzel PGJ et al (1992) Evaluation of the sensory irritation test for the assessment of occupational health risk. Crit RevToxicol 21:423-450

47. Korsak Z, Sokal J, Dedyk A et al (1988) Toxic effects of combined exposure to toluene and xylene in animals. I. Acute inhalation study. Pol J Occup Med 1:45-50

48. Olson BA, Gamberale F, Iregren A (1985) Coexposure to toluene and $p$-xylene in man: central nervous functions. Br J Ind Med 42:117-122
49. Dudek B, Gralewicz K, Jakubowski M et al (1990) Neurobehavioral effects of experimental exposure to toluene, xylene and their mixture. Pol J Occup Med 3:109-116

50. Gamberale F, Annwall G, Hultengren M (1978) Exposure to xylene and ethylbenzene. III. Effects on central nervous functions. Scand J Work Environ Health 4:204-211

51. Savolainen K, Linnavuo M (1979) Effects of mxylene on human equilibrium measured with a quantitative method. Acta Pharmacol Toxicol (Copenh) 44:315-318

52. Savolainen K, Riihimaki V, Vaheri E et al (1980) Effects of xylene and alcohol on vestibular and visual functions in man. Scand J Work Environ Health 6:94-103

53. Savolainen K, Riihimaki V, Laine A et al (1981) Short-term exposure of human subjects to $m-x y-$ lene and 1,1,1-trichloroethane. Int Arch Occup Environ Health 49:89-98

54. Savolainen K, Riihimaki V, Laine A (1982) Biphasic effects of inhaled solvents on human equilibrium. Acta Pharmacol Toxicol (Copenh) 51:237242

55. Savolainen K (1980) Combined effects of xylene and alcohol on the central nervous system. Acta Pharmacol Toxicol (Copenh) 46:366-372

56. Laine A, Savolainen K, Riihimaki V et al (1993) Acute effects of $m$-xylene inhalation on body sway, reaction times, and sleep in man. Int Arch Occup Environ Health 65:179-188

57. Savolainen K, Riihimaki V, Laine A et al (1982) Short-term exposure of human subjects to $\mathrm{m}$ xylene and 1,1,1-trichloroethane. Arch Toxicol Suppl 5:96-99

58. Savolainen K, Riihimaki V (1981) Xylene and alcohol involvement of the human equilibrium system. Acta Pharmacol Toxicol (Copenh) 49:447451

59. Savolainen K, Riihimaki V, Linnoila M (1979) Effects of short-term xylene exposure on psychophysiological functions in man. Int Arch Occup Environ Health 44:201-211

60. Savolainen K, Riihimaki V, Seppalainen AM et al (1980) Effects of short-term m-xylene exposure and physical exercise on the central nervous system. Int Arch Occup Environ Health 45:105-121

61. Hake CL, Stewart RD, Wu Sa et al (1981) p-Xylene: development of a biological standard for the industrial worker by breath analysis. PB82-152844. $\mathrm{NIOSH}$, by Medical College of Wisconsin, DoE, Milwaukee, WI. Zitiert nach NRC (2008)

62. Uchida Y, Nakatsuka H, Ukai H et al (1993) Symptoms and signs in workers exposed predominantly to xylenes. Int Arch Occup Environ Health 64:597-605

63. Chen Z, Liu SJ, Cai SX et al (1994) Exposure of workers to a mixture of toluene and xylenes. II. Effects. Occup Environ Med 51:47-49

64. Moser VC, Coggeshall EM, Balster RL (1985) Effects of xylene isomers on operant responding and motor performance in mice. Toxicol Appl Pharmacol 80:293-298

65. Molnar J, Paksy KA, Naray M (1986) Changes in the rat's motor behaviour during 4-hr inhalation exposure to prenarcotic concentrations of benzene and its derivatives. Acta Physiol Hung 67:349354

66. Korsak Z, Sokal JA, Wasiela T et al (1990) Toxic effects of acute exposure to particular xylene isomers in animals. Pol J Occup Med 3:221-226
67. Korsak Z, Swiercz R, Jedrychowski R (1993) Effects of acute combined exposure to $n$-butyl alcohol and m-xylene. Pol J Occup Med Environ Health 6:35-41

68. Korsak Z, Sokal JA, Gorny R (1992) Toxic effects of combined exposure to toluene and $m$-xylene in animals. III. Subchronic inhalation study. Pol J Occup Med Environ Health 5:27-33

69. Korsak Z, Wisniewska-Knypl J, Swiercz R (1994) Toxic effects of subchronic combined exposure to n-butyl alcohol and m-xylene in rats. Int J Occup Med Environ Health 7:155-166

70. Gralewicz S, Wiaderna D, Tomas T (1995) Development of spontaneous, age-related nonconvulsive seizure electrocortical activity and radialmaze learning after exposure to $m$-xylene in rats. Int J Occup Med Environ Health 8:347-360

71. Gralewicz S, Wiaderna D (2001) Behavioral effects following subacute inhalation exposure to $m-x y$ lene or trimethylbenzene in the rat: a comparative study. Neurotoxicology 22:79-89

72. Gagnaire F, Langlais C (2005) Relative ototoxicity of 21 aromatic solvents. Arch Toxicol 79:346-354

73. Maguin K, Lataye R, Campo P et al (2006) Ototoxicity of the three xylene isomers in the rat. Neurotoxicol Teratol 28:648-656

74. Gagnaire F, Marignac B, Langlais C et al (2001) Ototoxicity in rats exposed to ortho-, meta- and para-xylene vapours for 13 weeks. Pharmacol Toxicol 89:6-14

75. Gagnaire F, Langlais C, Grossmann S et al (2007) Ototoxicity in rats exposed to ethylbenzene and to two technical xylene vapours for 13 weeks. Arch Toxicol 81:127-143

76. Pryor GT, Rebert CS, Howd RA (1987) Hearing loss in rats caused by inhalation of mixed xylenes and styrene. J Appl Toxicol 7:55-61

77. Nylen P, Hagman M (1994) Function of the auditory and visual systems, and of peripheral nerve, in rats after long-term combined exposure to $\mathrm{n}$ hexane and methylated benzene derivatives. II. Xylene. Pharmacol Toxicol 74:124-129

78. Korsak Z, Rydzynski K (1994) Effects of acute combined inhalation exposure to n-butyl alcohol and n-butyl acetate in experimental animals. Int J Occup Med Environ Health 7:273-280

79. Chen CS, Hseu YC, Liang SH et al (2008) Assessment of genotoxicity of methyl-tert-butyl ether, benzene, toluene, ethylbenzene, and xylene to human lymphocytes using comet assay. J Hazard Mater 153:351-356

80. NAS/COT (2010) Acute Exposure Guideline Levels (AEGLs). Xylenes (CAS Reg. No. 1330-20-7). Acute Exposure Guideline Levels for SelectedAirborne Chemicals 9, Washington DC, USA S 293-380

81. Tatrai E, Ungvary G, Cseh IR et al (1981) The effect of long-term inhalation of o-xylene on the liver. Ind. Environ. Xenobiotics, Proc. Int. Conf, S 293 300. Zitiert nach US EPA (2003)

82. Maltoni C, Conti B, Cotti G et al (1985) Experimental studies on benzene carcinogenicity at the Bologna Institute of Oncology: current results and ongoing research. Am J Ind Med 7:415-446

83. NTP (1986) Toxicology and Carcinogenesis Studies of Xylenes (mixed) (CAS No. 1330-20-7) in F344/n Rats and B6C3F1 Mice (Gavage Studies). 327. U.S. Department of Health and Human Services, Public Health Services, National Institutes of Health, http://ntp.niehs.nih.gov/ntp/htdocs/ It_rpts/tr327.pdf 
84. Biodynamics I (1983) Parental and fetal reproduction inhalation toxicity study in rats with mixed xylene. Project \# 80-250. Prepared for U.S. EPA under TSCA, East Millstone, NJ. Zitiert nach US EPA (2003)

85. Nylen P, Ebendal T, Eriksdotter-Nilsson $\mathrm{M}$ et al (1989) Testicular atrophy and loss of nerve growth factor-immunoreactive germ cell line in rats exposed to $n$-hexane and a protective effect of simultaneous exposure to toluene or xylene. Arch Toxicol 63:296-307

86. Ungvary G, Tatrai E, Hudak A et al (1980) Studies on the embryotoxic effects of ortho-, meta- and para-xylene. Toxicology 18:61-74

87. Ungvary G, Tatrai E (1985) On the embryotoxic effects of benzene and its alkyl derivatives in mice, rats and rabbits. Arch Toxicol Suppl 8:425-430

88. Litton Bionetics (1978) Teratology study in rats xylene. Document 878210350 . EPA/OTS Public Files. Zitiert nach US EPA (2003), Washington DC, USA

89. Saillenfait AM, Gallissot F, Morel G et al (2003) Developmental toxicities of ethylbenzene, ortho-, meta-, para-xylene and technical xylene in rats following inhalation exposure. Food Chem Toxicol 41:415-429

90. DFG (2004) Xylol (alle Isomeren). Gesundheitsschädliche Arbeitsstoffe. Toxikologisch-arbeitsmedizinische Begründungen von MAK-Werten, 38. Lieferung. Wiley-VCH, Weinheim, S 1-3

91. Hass U, Lund SP, Simonsen L (1997) Long-lasting neurobehavioral effects of prenatal exposure to xylene in rats. Neurotoxicology 18:547-551

92. Hass U, Lund SP, Simonsen L et al (1995) Effects of prenatal exposure to xylene on postnatal development and behavior in rats. Neurotoxicol Teratol 17:341-349

93. Hass U, Jakobsen BM (1993) Prenatal toxicity of xylene inhalation in the rat: a teratogenicity and postnatal study. Pharmacol Toxicol 73:20-23

94. Rosen MB, Crofton KM, Chernoff N (1986) Postnatal evaluation of prenatal exposure to $p$-xylene in the rat. Toxicol Lett 34:223-229

95. Nagata Y (2003) Measurement of odor threshold by triangle odor bag method. Odor Measurement Review. Japan. Ministry of the Environment, http://www.env.go.jp/en/air/odor/measure/02_3_2.pdf

96. AGS (2014) TRGS 900 „Arbeitsplatzgrenzwerte". GMBL. Bundesministerium des Innern (BMI), http://www.baua.de/de/Themen-von-A-Z/Gefahrstoffe/TRGS/TRGS-900.htm

97. Pflaumbaum W, Bagschick U, Blome $\mathrm{H}$ et al (2008) Neue Arbeitsplatzgrenzwerte für Kohlenwasserstoffgemische (Lösemittelkohlenwasserstoffe). Teil 1: Ableitung und Anwendung. Gefahrstoffe, Reinhaltung der Luft 6:270-274

98. Baars AJ, Theelen RMC, Janssen PJCM et al (2001) Re-evaluation of human-toxicological maximum permissible risk levels. RIVM Report No. 711701025. National Institute of Public Health and the Environment (RIVM), Bilthoven

99. U.S.EPA (2003) IRIS Substance file - Xylenes, CASRN 1330-20-7. Integrated Risk Information System (IRIS). U.S. Environmental Protection Agency, Washington, D.C. http://www.epa.gov/ iris/subst/0270.htm

100. Schupp T, Bolt HM, Hengstler JG (2005) Maximum exposure levels for xylene, formaldehyde and acetaldehyde in cars. Toxicology 206:461470
101. Ad-hoc-Arbeitsgruppe Innenraumrichtwerte der IRK/AGLMB (2012) Richtwerte für die Innenraumluft: erste Fortschreibung des Basisschemas. Bundesgesundheitsblatt 55:279-290 